\title{
Melena: a rare presentation of childhood Burkitt's lymphoma
}

THE CASE: A 10-year-old previously well boy presented with a 5-day history of constant epigastric abdominal pain not associated with meals. He had had 3 melena stools and 2 episodes of nonbilious, non-bloody emesis. He reported no NSAID use, weight loss or fever and had no history of excessive bleeding or of gastrointestinal or liver disease.

The patient was pale, afebrile and hemodynamically stable. His abdomen was soft and diffusely tender, with no rebound tenderness or guarding. There was no lymphadenopathy, hepatosplenomegaly or abdominal mass. A complete blood count showed a hemoglobin concentration of 80 (normal 120-160) g/L and a normal platelet count. The international normalized ratio, partial thromboplastin time, electrolyte levels, and renal and liver function test results were normal.

Upper endoscopy revealed multiple ulcerated masses in the stomach, duodenum and jejunum that were sug-

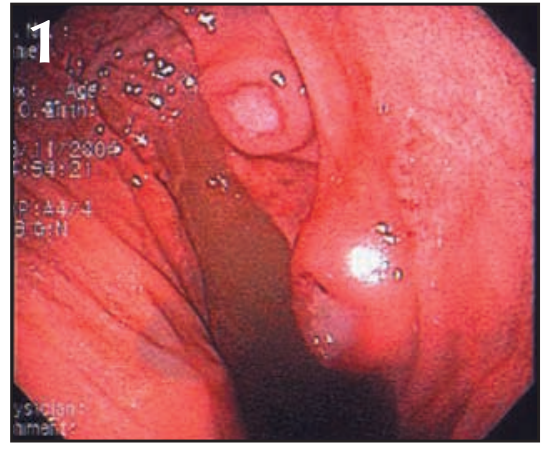
gestive of cancer (Fig. 1). CT scans of the chest and abdomen showed multiple lesions in the liver, kidneys, pancreas, stomach and small bowel (Fig. 2 and Fig. 3) and significantly enlarged lymph nodes in the retroperitoneum, crural area and inguinal canal. Immunohistochemical staining of the biopsied gastric lesions identified Burkitt's lymphoma. The patient was initially given a proton pump inhibitor intravenously and then combination chemotherapy. He responded rapidly and remains in remission 1 year after completing the treatment.
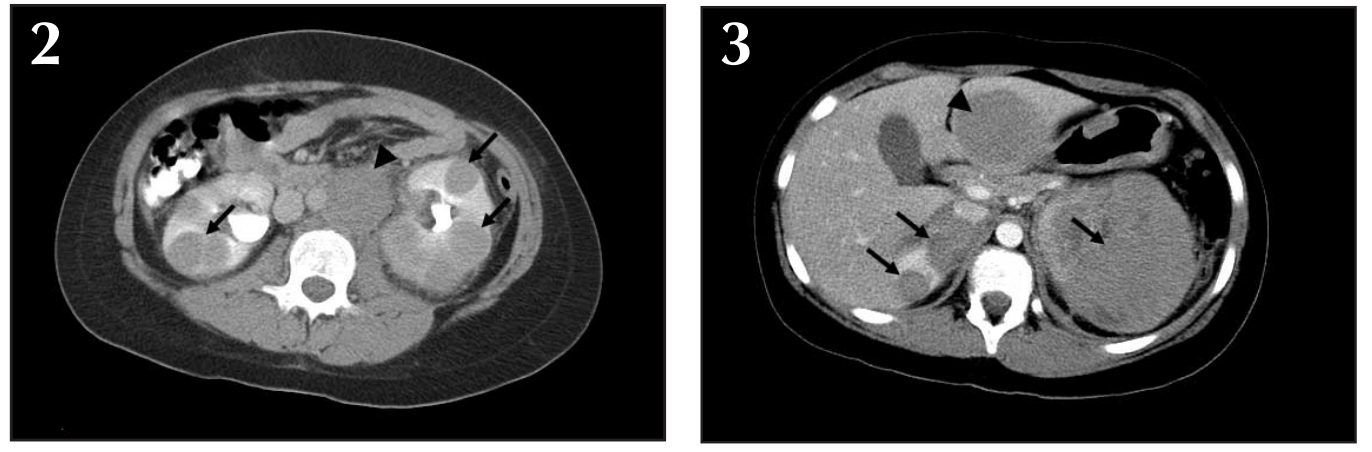

$\mathrm{T}$ he presentation of melena and anemia indicates bleeding in the upper gastrointestinal tract. In children (other than newborns) who have a history of abdominal pain and no evidence of portal hypertension, the most common causes of such bleeding include gastritis and peptic ulcer disease, which - can be associated with NSAID or salicylate use, Helicobacter pylori infection or severe systemic illness (Box 1). ${ }^{1}$ Lacerations associated with Mallory-Weiss syndrome may occur following persistent vomiting. Esophagitis is possible in children with se- vere gastroesophageal reflux disease, which is often associated with neuromuscular disease or hiatal hernia or can be due to ingestion of a caustic substance or to infection. Less common causes of bleeding in the upper gastrointestinal tract include bleeding disorders, ZollingerEllison syndrome, hemangiomatous malformations, gastrointestinal duplications and malignant disease. ${ }^{1}$ Early endoscopy helps to identify the cause of bleeding and may enable the use of endoscopic therapies to minimize the risk of recurrent bleeding. ${ }^{1}$
Non-Hodgkin's lymphoma and Hodgkin's lymphoma constitute $60 \%$ and $40 \%$ respectively of the pediatric lymphomas, which are the third most common type of malignant disease in North American children. ${ }^{2}$ Burkitt's lymphoma is the most common subtype of nonHodgkin's lymphoma, with an incidence of 2 cases per million children in North America (sporadic variety) and up to 100 cases per million children in equatorial Africa (endemic variety). ${ }^{3}$ It is believed that the Epstein-Barr virus plays a causal role, particularly in Africa. Burkitt's lym- 
phoma is characterized by a clonal proliferation of relatively mature $\mathrm{B}$ cells, and the majority of cases contain a chromosomal translocation - $\mathrm{t}(8 ; 14)$ - that leads to uncontrolled expression of a proto-oncogene.

Endemic Burkitt's lymphoma has a peak incidence among children 11 years old, usually presenting with the classic jaw tumour and occasionally involving the gastrointestinal tract and

Box 1: Causes of upper gastrointestinal tract bleeding in childhood

Neonates

- Ingested maternal blood

- Esophagitis

- Gastritis

- Gastroduodenal ulceration

- Allergy to cow's milk protein

- Coagulopathy (including hemorrhagic disease of the newborn)

- Anatomic and vascular anomalies

Older children

- Gastritis

- Esophagitis

- Gastroduodenal ulceration (from severe systemic illness, NSAID use or Helicobacter pylori infection)

- Varices

- Lacerations associated with Mallory-Weiss syndrome

- Epistaxis

- Foreign body

- Anatomic and vascular anomalies

- Coagulopathy

- Vasculitis

- Malignant disease

- Zollinger-Ellison syndrome kidneys. This is in contrast to the sporadic type, whose peak incidence is among children 7 years old and whose presentation largely involves the gastrointestinal tract. This results from direct involvement of Peyer's patches, and affected children commonly present with an abdominal mass or pain, distention, nausea and vomiting. Intussusception, obstruction or perforation may occur. ${ }^{4}$ However, presentation with bleeding in the upper gastrointestinal tract, as in the case we have described, has not been previously reported to our knowledge. Other common potential sites affected by the sporadic form of Burkitt's lymphoma include tonsils or adenoids, bone marrow, bone, kidneys, testes, the central nervous system and salivary glands. Both forms of Burkitt's lymphoma occur more often in boys than in girls, at a ratio of 2-3:1.

Rapid tumour growth, with a doubling time of $2-3$ days, is one of the features of the disease and necessitates urgent diagnostic evaluation. A high rate of spontaneous cell death results in tumour lysis syndrome, which consists of potentially fatal electrolyte abnormalities such as hyperkalemia, hyperuricemia, hyperphosphatemia and hypocalcemia, as well as oliguric renal failure owing to uric acid nephropathy. Because of the tumour's rapid doubling time, two-thirds of patients have extensive disease involving bone marrow or the central nervous system, or both, at presentation. Therefore, after the initial surgical biopsy, combination chemotherapy (varying in intensity and duration relative to the extent of the presenting disease) and vigilant precautions against tumour lysis (e.g., vigorous hydration) is the treatment of choice. Survival rates now approach $90 \%-100 \%$ among patients with limited, localized disease and $75 \%-85 \%$ among those with extensive disease. ${ }^{4}$

\section{Joanna Holland}

Michaela Cada

Department of Pediatrics

University of Toronto

Simon C. Ling

Division of Gastroenterology,

Hepatology and Nutrition

Michael L. Capra

Division of Haematology/Oncology

Stacey Bernstein

Division of Pediatric Medicine

Hospital for Sick Children

University of Toronto

Toronto, Ont.

\section{References}

1. Fox VL. Gastrointestinal bleeding in infancy and childhood. Gastroenterol Clin North Am 2000;29:37-66.

2. Sandlund JT, Downing JR, Crist WM. Non-Hodgkin's lymphoma in childhood. $N$ Engl 7 Med 1996;334: 1238-48.

3. Parkin DM, Stiller CA, Draper GJ, Bieber CA. The international incidence of childhood cancer. Int 7 Cancer 1988;42:511-20.

4. Shad A, Magrath I. Non-Hodgkin's lymphoma. Pediatr Clin North Am 1997;44:863-90. 